\title{
Electron correlations in narrow energy bands: ground state energy and metal-insulator transition
}

\author{
L.Didukh* Yu.Skorenkyy \\ Ternopil State Technical University, Department of Physics, \\ 56 Rus'ka Str., 46001 Ternopil, Ukraine \\ Received May 5, 2000, in final form October 28, 2000
}

\begin{abstract}
The electron correlations in narrow energy bands are examined within the framework of the Hubbard model. The single-particle Green function and energy spectrum are obtained in a paramagnetic state at half-filling by means of a new two-pole approximation. Analytical expressions for the energy gap, polar states concentration and energy of the system are found in the ground state. Metal-insulator transitions in the model at the change of bandwidth or temperature are investigated. The results obtained are used for interpretation of some experimental data in narrow-band materials.
\end{abstract}

PACS: $71.28 .+d, 71.27 .+a, 71.10 . F d, 71.30 .+h$

Key words: narrow energy bands, Hubbard Hamiltonian, metal-insulator transition, ground state energy

\section{Introduction}

Among the metal-insulator transitions (MIT) observed in narrow-band materials of great interest are the transitions from paramagnetic metal state to paramagnetic insulator state at the increase of temperature exhibited by the systems $\mathrm{NiS}_{2-x} \mathrm{Se}_{x}[1-$ 3], $\left(\mathrm{V}_{1-x} \mathrm{Cr}_{x}\right)_{2} \mathrm{O}_{3}[1,4]$ and $\mathrm{Y}_{1-x} \mathrm{Ca}_{x} \mathrm{TiO}_{3}[5,6]$; in these systems the paramagnetic insulator - paramagnetic metal transitions under external pressure are observed as well. There are reasons to believe that the mentioned transitions are caused by the electron-electron interactions and can be described within the framework of Hubbard model [7].

Hubbard model is the simplest model describing MIT in the materials with narrow energy bands. This model describes a single non-degenerate band of electrons with the local Coulomb interaction. The model Hamiltonian contains two energy parameters: the hopping integral of an electron from one site to another and the

*E-mail: didukh@tu.edu.te.ua, Tel.:+380352251946, Fax: +380352254983 
intraatomic Coulomb repulsion of two electrons of the opposite spins. This model is used intensively (for recent reviews see $[1,8-10]$ ) in order to describe the peculiarities of physical properties of narrow-band materials; in this connection two-pole approaches seem to be attractive. The two-pole approaches in the Hubbard model and the Hubbard bands conception (being caused by the two-pole approximation) have been useful in understanding the peculiarities of electric and magnetic properties of narrow-band materials $[1,10]$. However within the framework of two-pole approaches there is a number of issues, in particular the problem of describing metal-insulator transitions [10,14-21].

In the present paper, the recently proposed two-pole approximation [11] is used to study the effects of electron correlations in the Hubbard model. The single particle Green function and the energy spectrum are obtained. Analytical dependencies of the energy gap, polar states (doublons or holes) concentration and the energy of the system on the model parameters are found in the ground state. Dependencies for the energy gap, polar states concentration on temperature are calculated. The results obtained are compared with the corresponding results of other approximations and are used for the interpretation of some experimental data. In particular, the observable transitions from an insulating state to a metallic state at the increase of a bandwidth, and from a metallic state to an insulating state at the increasing temperature are explained.

\section{Single-particle Green function and energy spectrum}

The Hubbard Hamiltonian [1] in terms of transition-operators of $i$-site from state $|l\rangle$ to state $|k\rangle X_{i}^{k l}[12]$ is written as

$$
\begin{aligned}
H & =H_{0}+H_{1}+H_{1}^{\prime} \\
H_{0} & =-\mu \sum_{i \sigma}\left(X_{i}^{\sigma}+X_{i}^{2}\right)+U \sum_{i} X_{i}^{2}, \\
H_{1} & =\sum_{i j \sigma, i \neq j} t_{i j}\left(X_{i}^{\sigma 0} X_{j}^{0 \sigma}+X_{i}^{2 \bar{\sigma}} X_{j}^{\bar{\sigma} 2}\right), \\
H_{1}^{\prime} & =\sum_{i j, i \neq j} t_{i j}\left(X_{i}^{\bar{\sigma} 0} X_{j}^{\sigma 2}-X_{i}^{\sigma 0} X_{j}^{\bar{\sigma} 2}+\text { h.c. }\right),
\end{aligned}
$$

where $\mu$ is the chemical potential, $U$ is the intra-atomic Coulomb repulsion, $t_{i j}$ is the nearest-neighbour hopping integral, $X_{i}^{k}$ is the operator of the number of $|k\rangle$-states on $i$-site; $\sigma$ denotes the spin of an electron $(\sigma=\downarrow, \uparrow)$ and $\bar{\sigma}$ denotes the projection of an electron spin opposite to $\sigma ; H_{0}$ describes a system in the atomic limit, $H_{1}$ describes electron hoppings between singly occupied sites and empty sites (holes) (the first sum in $H_{1}$ - processes forming "h-band") and electron hoppings between doubly occupied sites (doublons) and singly occupied sites (the second sum in $H_{1}$ - processes forming "d-band"). $H_{1}^{\prime}$ describes "hybridization" between the "h-band" and "d-band" (the processes of pair creation and annihilation of holes and doublons). 
The single-particle Green function is written in $X_{i}^{k l}$-operators as

$$
\left\langle\left\langle a_{p \uparrow} \mid a_{s \uparrow}^{+}\right\rangle\right\rangle=\left\langle\left\langle X_{p}^{\downarrow 2} \mid X_{s}^{2 \downarrow}\right\rangle\right\rangle-\left\langle\left\langle X_{p}^{0 \uparrow} \mid X_{s}^{2 \downarrow}\right\rangle\right\rangle-\left\langle\left\langle X_{p}^{\downarrow 2} \mid X_{s}^{\uparrow 0}\right\rangle\right\rangle+\left\langle\left\langle X_{p}^{0 \uparrow} \mid X_{s}^{\uparrow 0}\right\rangle\right\rangle .
$$

The functions $\left\langle\left\langle X_{p}^{\downarrow 2} \mid X_{s}^{2 \downarrow}\right\rangle\right\rangle$ and $\left\langle\left\langle X_{p}^{0 \uparrow} \mid X_{s}^{2 \downarrow}\right\rangle\right\rangle$ satisfy the equations

$$
\begin{aligned}
(E+\mu-U)\left\langle\left\langle X_{p}^{\downarrow 2} \mid X_{s}^{2 \downarrow}\right\rangle\right\rangle= & \frac{\delta_{p s}}{2 \pi}\left\langle X_{p}^{\downarrow}+X_{p}^{2}\right\rangle+\left\langle\left\langle\left[X_{p}^{\downarrow 2}, H_{1}\right]_{-} \mid X_{s}^{2 \downarrow}\right\rangle\right\rangle \\
& +\left\langle\left\langle\left[X_{p}^{\downarrow 2}, H_{1}^{\prime}\right]_{-} \mid X_{s}^{2 \downarrow}\right\rangle\right\rangle, \\
(E+\mu)\left\langle\left\langle X_{p}^{0 \uparrow} \mid X_{s}^{2 \downarrow}\right\rangle\right\rangle= & \left\langle\left\langle\left[X_{p}^{0 \uparrow}, H_{1}\right]_{-} \mid X_{s}^{2 \downarrow}\right\rangle\right\rangle+\left\langle\left\langle\left[X_{p}^{0 \uparrow}, H_{1}^{\prime}\right]_{-} \mid X_{s}^{2 \downarrow}\right\rangle\right\rangle,
\end{aligned}
$$

with $[A, B]_{-}=A B-B A$. To obtain the closed system of equations we apply a new two-pole approximation, proposed in the work [11]. Suppose in equation (2.6) that

$$
\begin{aligned}
{\left[X_{p}^{0 \uparrow}, H_{1}\right]_{-} } & =\sum_{j} \epsilon(p j) X_{j}^{0 \uparrow}, \\
{\left[X_{p}^{\downarrow 2}, H_{1}\right]_{-} } & =\sum_{j} \tilde{\epsilon}(p j) X_{j}^{\downarrow 2},
\end{aligned}
$$

where $\epsilon(p j)$ and $\tilde{\epsilon}(p j)$ are non-operator expressions which we calculate using the method of the work [13]. In the considered model, in the particular case of electron concentration $n=1$ in a paramagnetic state we have

$$
\epsilon(p j)=\tilde{\epsilon}(p j)=(1-2 d) t_{p j}
$$

with $d=\left\langle X_{p}^{2}\right\rangle$ being the concentration of doublons.

Let us take into account the functions $\left\langle\left\langle\left[X_{p}^{\downarrow 2}, H_{1}^{\prime}\right]_{-} \mid X_{s}^{2 \downarrow}\right\rangle\right\rangle$ and $\left\langle\left\langle\left[X_{p}^{0 \uparrow}, H_{1}^{\prime}\right]_{-} \mid X_{s}^{2 \downarrow}\right\rangle\right\rangle$ in Hartree-Fock approximation:

$$
\begin{aligned}
& \left\langle\left\langle\left[X_{p}^{\downarrow 2}, H_{1}^{\prime}\right]_{-} \mid X_{s}^{2 \downarrow}\right\rangle\right\rangle= \\
& =-\sum_{i, i \neq p} t_{i p}\left[\left\langle\left\langle\left(X_{p}^{\downarrow}+X_{p}^{2}\right) X_{i}^{0 \uparrow} \mid X_{s}^{2 \downarrow}\right\rangle\right\rangle+\left\langle\left\langle X_{p}^{02} X_{i}^{2 \uparrow} \mid X_{s}^{2 \downarrow}\right\rangle\right\rangle-\left\langle\left\langle X_{p}^{\downarrow \uparrow} X_{i}^{0 \downarrow} \mid X_{s}^{2 \downarrow}\right\rangle\right\rangle\right] \\
& \simeq-\sum_{i, i \neq p} t_{i p}\left\langle X_{p}^{\downarrow}+X_{p}^{2}\right\rangle\left\langle\left\langle X_{i}^{0 \uparrow} \mid X_{s}^{2 \downarrow}\right\rangle\right\rangle, \\
& \left\langle\left\langle\left[X_{p}^{0 \uparrow}, H_{1}^{\prime}\right]_{-} \mid X_{s}^{2 \downarrow}\right\rangle\right\rangle= \\
& =-\sum_{i, i \neq p} t_{i p}\left[\left\langle\left\langle\left(X_{p}^{0}+X_{p}^{\uparrow}\right) X_{i}^{\downarrow 2} \mid X_{s}^{2 \downarrow}\right\rangle\right\rangle+\left\langle\left\langle X_{p}^{02} X_{i}^{\downarrow 0} \mid X_{s}^{2 \downarrow}\right\rangle\right\rangle-\left\langle\left\langle X_{p}^{\downarrow \uparrow} X_{i}^{\uparrow 2} \mid X_{s}^{2 \downarrow}\right\rangle\right\rangle\right] \\
& \simeq-\sum_{i, i \neq p} t_{i p}\left\langle X_{p}^{0}+X_{p}^{\uparrow}\right\rangle\left\langle\left\langle X_{i}^{\downarrow 2} \mid X_{s}^{2 \downarrow}\right\rangle\right\rangle ;
\end{aligned}
$$

this way we neglect the processes describing the "inter-band" hoppings of electrons which are connected with the spin turning over and the "inter-band" hoppings with the formation or annihilation of two electrons on the same site. 
So we obtain the closed system of equations

$$
\begin{aligned}
&(E-\mu+U)\left\langle\left\langle X_{p}^{\downarrow 2} \mid X_{s}^{2 \downarrow}\right\rangle\right\rangle-\sum_{i} \tilde{\epsilon}(p i)\left\langle\left\langle X_{i}^{\downarrow 2} \mid X_{s}^{2 \downarrow}\right\rangle\right\rangle \\
&+\left\langle X_{p}^{\downarrow}+X_{p}^{2}\right\rangle \sum_{i, i \neq p} t_{i p}\left\langle\left\langle X_{i}^{\downarrow 2} \mid X_{s}^{2 \downarrow}\right\rangle\right\rangle=\frac{\left\langle X_{p}^{2}+X_{p}^{\downarrow}\right\rangle}{2 \pi} \delta_{p s}, \\
&(E-\mu)\left\langle\left\langle X_{p}^{0 \uparrow} \mid X_{s}^{2 \downarrow}\right\rangle\right\rangle-\sum_{i} \epsilon(p i)\left\langle\left\langle X_{i}^{0 \uparrow} \mid X_{s}^{2 \downarrow}\right\rangle\right\rangle \\
&+\left\langle X_{p}^{0}+X_{p}^{\uparrow}\right\rangle \sum_{i, i \neq p} t_{i p}\left\langle\left\langle X_{i}^{\downarrow 2} \mid X_{s}^{2 \downarrow}\right\rangle\right\rangle=0 .
\end{aligned}
$$

After the Fourier transformation we obtain solutions of the system of equations (2.10)

$$
\begin{aligned}
\left\langle\left\langle X_{p}^{\downarrow 2} \mid X_{s}^{2 \downarrow}\right\rangle\right\rangle_{\mathbf{k}}= & \frac{\left\langle X_{p}^{2}+X_{p}^{\downarrow}\right\rangle}{2 \pi}\left(\frac{A_{\mathbf{k}}^{1}}{E-E_{h}(\mathbf{k})}+\frac{B_{\mathbf{k}}^{1}}{E-E_{d}(\mathbf{k})}\right), \\
A_{\mathbf{k}}^{1}= & \frac{1}{2}\left(1-\frac{U-\epsilon(\mathbf{k})+\tilde{\epsilon}(\mathbf{k})}{E_{d}(\mathbf{k})-E_{h}(\mathbf{k})}\right), \quad B_{\mathbf{k}}^{1}=1-A_{\mathbf{k}}^{1}, \\
\left\langle\left\langle X_{p}^{0 \uparrow} \mid X_{s}^{2 \downarrow}\right\rangle\right\rangle_{\mathbf{k}}= & \frac{\left\langle X_{p}^{2}+X_{p}^{\downarrow}\right\rangle\left\langle X_{p}^{0}+X_{p}^{\uparrow}\right\rangle}{2 \pi} \\
& \times \frac{t(\mathbf{k})}{E_{d}(\mathbf{k})-E_{h}(\mathbf{k})}\left(\frac{1}{E-E_{h}(\mathbf{k})}-\frac{1}{E-E_{d}(\mathbf{k})}\right) .
\end{aligned}
$$

Here $t(\mathbf{k})$ is the hopping integral in $\mathbf{k}$-representation and

$$
\begin{aligned}
E_{h}(\mathbf{k})= & -\mu+\frac{U}{2}+\frac{\epsilon(\mathbf{k})+\tilde{\epsilon}(\mathbf{k})}{2} \\
& -\frac{1}{2} \sqrt{[U-\epsilon(\mathbf{k})+\tilde{\epsilon}(\mathbf{k})]^{2}+\left\langle X_{p}^{0}+X_{p}^{\uparrow}\right\rangle\left\langle X_{p}^{\downarrow}+X_{p}^{2}\right\rangle(t(\mathbf{k}))^{2}}, \\
E_{d}(\mathbf{k})= & -\mu+\frac{U}{2}+\frac{\epsilon(\mathbf{k})+\tilde{\epsilon}(\mathbf{k})}{2} \\
& +\frac{1}{2} \sqrt{[U-\epsilon(\mathbf{k})+\tilde{\epsilon}(\mathbf{k})]^{2}+\left\langle X_{p}^{0}+X_{p}^{\uparrow}\right\rangle\left\langle X_{p}^{\downarrow}+X_{p}^{2}\right\rangle(t(\mathbf{k}))^{2}}
\end{aligned}
$$

are the energies of electron in lower ("hole") and upper ("doublon") subbands, respectively; $\epsilon(\mathbf{k})$ and $\tilde{\epsilon}(\mathbf{k})$ are the Fourier components of $\epsilon(p j)$ and $\tilde{\epsilon}(p j)$.

An analogous procedure for functions $\left\langle\left\langle X_{p}^{\downarrow 2} \mid X_{s}^{\uparrow 0}\right\rangle\right\rangle$ and $\left\langle\left\langle X_{p}^{0 \uparrow} \mid X_{s}^{\uparrow 0}\right\rangle\right\rangle$ gives the following expressions:

$$
\begin{aligned}
\left\langle\left\langle X_{p}^{\downarrow 2} \mid X_{s}^{\uparrow 0}\right\rangle\right\rangle_{\mathbf{k}} & =\left\langle\left\langle X_{p}^{0 \uparrow} \mid X_{s}^{2 \downarrow}\right\rangle\right\rangle_{\mathbf{k}}, \\
\left\langle\left\langle X_{p}^{0 \uparrow} \mid X_{s}^{\uparrow 0}\right\rangle\right\rangle_{\mathbf{k}} & =\frac{\left\langle X_{p}^{0}+X_{p}^{\uparrow}\right\rangle}{2 \pi}\left(\frac{A_{\mathbf{k}}^{2}}{E-E_{h}(\mathbf{k})}-\frac{B_{\mathbf{k}}^{2}}{E-E_{d}(\mathbf{k})}\right), \\
A_{\mathbf{k}}^{2} & =B_{\mathbf{k}}^{1}, \quad B_{\mathbf{k}}^{2}=A_{\mathbf{k}}^{1} .
\end{aligned}
$$

Finally, in k-representation single-particle Green function (2.5) we obtain

$$
\left\langle\left\langle a_{p \uparrow} \mid a_{s \uparrow}^{+}\right\rangle\right\rangle_{\mathbf{k}}=\frac{1}{2 \pi}\left(\frac{A_{\mathbf{k}}}{E-E_{h}(\mathbf{k})}+\frac{B_{\mathbf{k}}}{E-E_{d}(\mathbf{k})}\right),
$$




$$
\begin{aligned}
& A_{\mathbf{k}}=\frac{1}{2}\left(1-\frac{\left(C_{1}-C_{2}\right)(U-\epsilon(\mathbf{k})+\tilde{\epsilon}(\mathbf{k}))+4 t(\mathbf{k}) C_{1} C_{2}}{E_{d}(\mathbf{k})-E_{h}(\mathbf{k})}\right), \\
& B_{\mathbf{k}}=1-A_{\mathbf{k}},
\end{aligned}
$$

where $C_{1}=\left\langle X_{p}^{0}+X_{p}^{\uparrow}\right\rangle, C_{2}=\left\langle X_{p}^{2}+X_{p}^{\downarrow}\right\rangle$.

To calculate the single-particle Green function and energy spectrum we substitute the mean values of diagonal $X$-operators by their mean values:

$$
\left\langle X_{p}^{2}\right\rangle=d, \quad\left\langle X_{p}^{0}\right\rangle=c .
$$

In an important case for the investigation of metal-insulator transition $n=1$ (when $c=d$ ) in a paramagnetic state $\left(\left\langle X_{p}^{\uparrow}\right\rangle=\left\langle X_{p}^{\downarrow}\right\rangle\right.$ ) from the constraint

$$
\left\langle X_{p}^{0}+X_{p}^{2}+X_{p}^{\uparrow}+X_{p}^{\downarrow}\right\rangle=1
$$

we have the following:

$$
\left\langle X_{p}^{\uparrow}\right\rangle=\left\langle X_{p}^{\downarrow}\right\rangle=\frac{1}{2}(1-2 d) .
$$

Finally, single-particle Green function (2.16) has the form

$$
\begin{aligned}
& \left\langle\left\langle a_{p \uparrow} \mid a_{s \uparrow}^{+}\right\rangle\right\rangle_{\mathbf{k}}=\frac{1}{2 \pi}\left(\frac{A_{\mathbf{k}}}{E-E_{h}(\mathbf{k})}+\frac{B_{\mathbf{k}}}{E-E_{d}(\mathbf{k})}\right), \\
& A_{\mathbf{k}}=\frac{1}{2}\left(1-\frac{t(\mathbf{k})}{\sqrt{U^{2}+(t(\mathbf{k}))^{2}}}\right), \\
& B_{\mathbf{k}}=1-A_{\mathbf{k}},
\end{aligned}
$$

where single-particle energy spectrum is

$$
\begin{aligned}
& E_{h}(\mathbf{k})=(1-2 d) t(\mathbf{k})-\frac{1}{2} \sqrt{U^{2}+(t(\mathbf{k}))^{2}}, \\
& E_{d}(\mathbf{k})=(1-2 d) t(\mathbf{k})+\frac{1}{2} \sqrt{U^{2}+(t(\mathbf{k}))^{2}}
\end{aligned}
$$

(here we took into account that $\mu=U / 2$ for $n=1$ ).

Single-particle Green function (2.20) and energy spectrum (2.21) are exact in the band and atomic limits. It is worthwile to note that unlike the results of two-pole approximations of Hubbard [7] and Ikeda, Larsen, Mattuck [14], the energy spectrum (2.21) depends on polar states concentration (thus on temperature). Unlike the approximations based on the ideology of Roth [15] (in this connection see also [16-21]), the energy spectrum (2.21) describes a metal-insulator transition. Energy spectrum which describes a metal-insulator transition was earlier obtained in work [13]. Unlike the work [13] where all commutators in the equations (2.6) were taken into account in the generalized mean-field approximation, here we apply this procedure only to the commutators of electron operators with the "diagonal" part of Hamiltonian which describes hoppings within Hubbard subbands. Other processes are 
taken into account by means of Hartree-Fock approximation. As a result, expression (2.21) differs from the respective expressions in work [13] by the presence of the term $\sqrt{U^{2}+t^{2}(\mathbf{k})}$ instead of $\sqrt{U^{2}+4 d^{2} t^{2}(\mathbf{k})}$. This leads to the series of distinctions between the results of this work and the results of work [13] $(d(U / w)$-dependence, the condition of metal-insulator transition, etc); at the same time expression (2.21) depends on polar state concentration similar to the respective expression in work [13].

\section{Energy gap and polar states concentration}

The energy gap (difference of energies between bottom of the upper and top of the lower Hubbard bands) is given by

$$
\Delta E=E_{d}(-w)-E_{h}(w)=-2 w(1-2 d)+\sqrt{U^{2}+w^{2}}
$$

(where $w=z|t|$ is the halfwidth of the uncorrelated electron band, $z$ is the number of the nearest neighbours to a site). Expression (3.1) describes the vanishing of the energy gap in the spectrum of paramagnetic insulator at critical value $(U / w)_{\text {c }}$ when the halfbandwidth $w$ increases (under pressure).

To calculate the polar states concentration we use the function (2.11). At $T=0$ and at a rectangular density of states, the concentration of polar states is

$$
d=\frac{1}{4}+\frac{U}{8 w} \ln \left(\frac{1-4 d}{3-4 d}\right)
$$

if $(U / w) \leqslant(U / w)_{\mathrm{c}}$ and

$$
d=\frac{1}{4}+\frac{U}{8 w} \ln \left(\frac{\sqrt{1+(U / w)^{2}}+1}{\sqrt{1+(U / w)^{2}}-1}\right)
$$

if $(U / w)>(U / w)_{\mathrm{c}}$. At $T=0$ we have $(U / w)_{\mathrm{c}}=1.672$.

The dependence $d(U / w)$ given by equations (3.2)-(3.3) is plotted on figure 1 . One can see that in the point $(U / w)_{\mathrm{c}}$ the slope of $d(U / w)$-dependence changes; the concentration of doublons vanishes at $U / w \rightarrow \infty$. Our result for $d(U / w)$ in the region of MIT is in good agreement with the result of papers $[9,23]$ obtained in the limit of infinite dimensions (figure 2). The parameter $U$ is normalized by the averaged band energy in the absence of correlation $\varepsilon_{0}$ (we have $\varepsilon_{0}=-w / 2$ when the density of states is rectangular). In figure 3 the dependencies

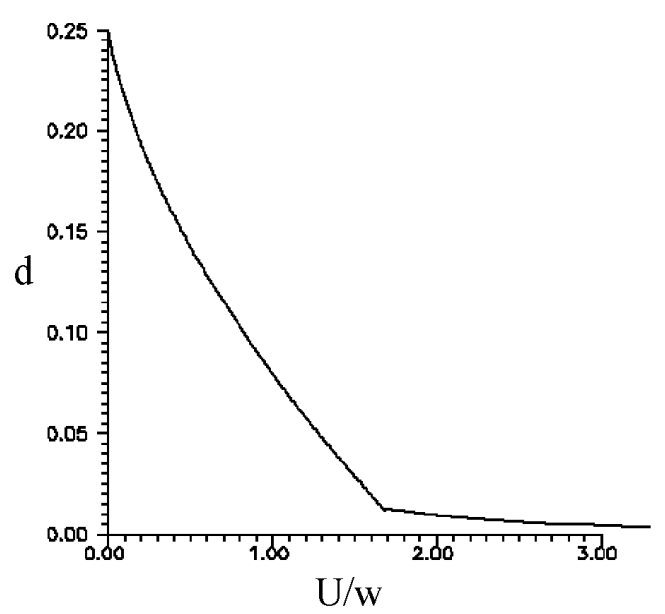

Figure 1. The dependence of doublon concentration $d$ on $U / w$ at zero temperature. 


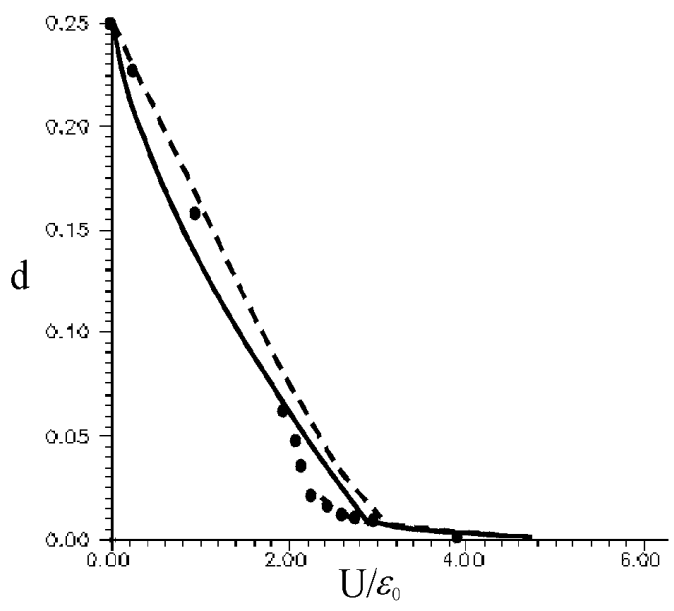

Figure 2. The comparison of $d(U / w)$ dependencies: solid line our result, dashed line - iterativeperturbative theory $[9,23]$, circles QMC method [23].

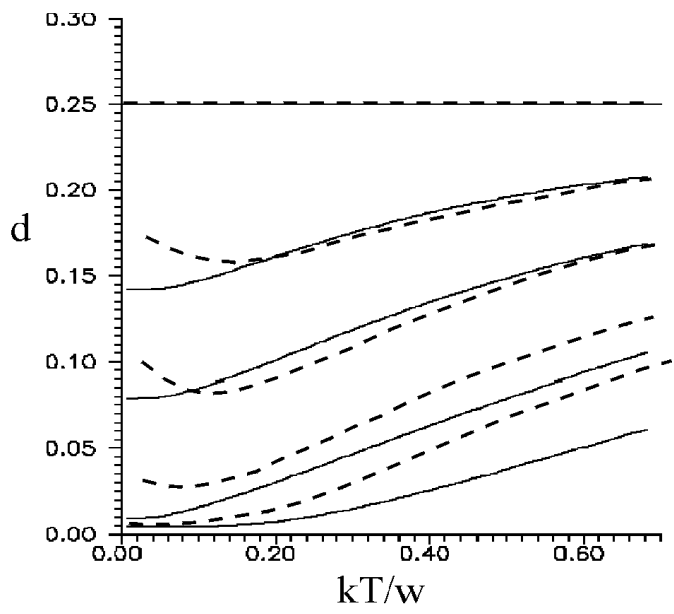

Figure 4. The dependencies of doublon concentration $d$ on temperature at different $U / w$ : values of $U / w$ from down to up are 2, 1.5, 1, 0.5, 0; solid lines correspond to our results, dashed lines correspond to the result of papers $[9,23]$.

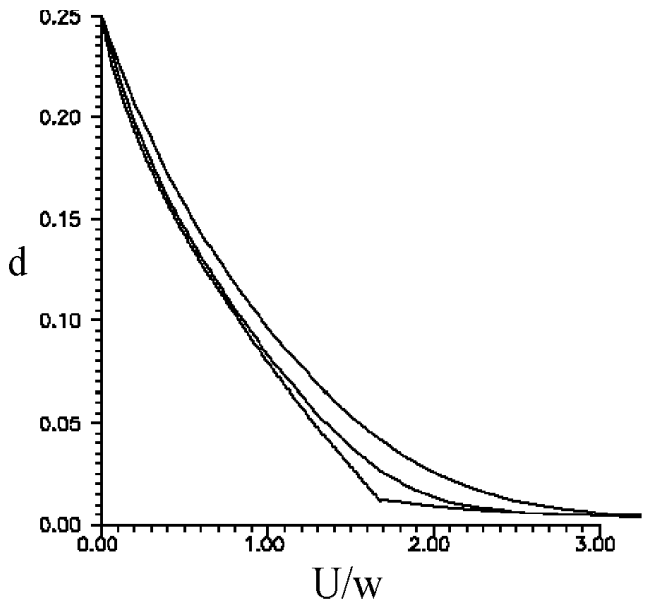

Figure 3. The dependencies of doublon concentration $d$ on $U / w$ at different temperatures: lower curve corresponds to $k T / w=0.16$, middle curve corresponds to $k T / w=0.08$, upper curve corresponds to $k T / w=0$.

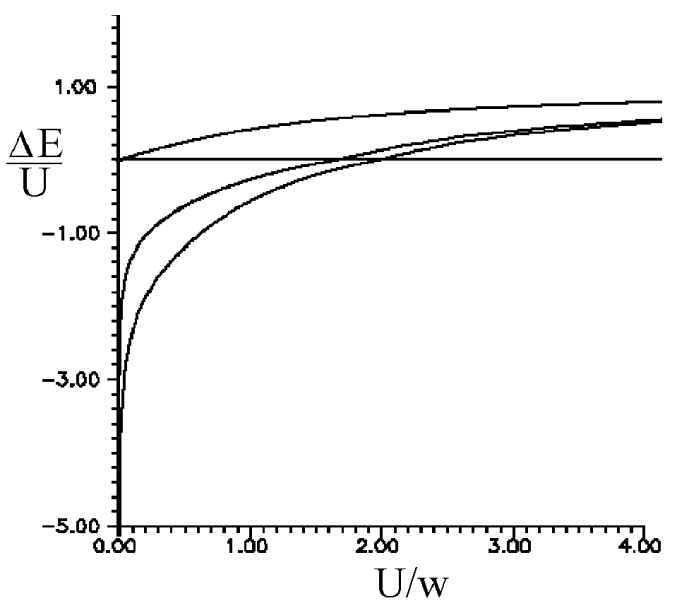

Figure 5. The dependencies of energy gap width on $U / w$ : "Hubbard-I" approximation (upper curve), our result (middle curve), approximation [13] (lower curve). 
of polar states concentration on parameter $U / w$ at different temperatures are presented. Note the important difference (see figure 4) of the dependence of $d$ on temperature from the result of papers $[9,23]$ : we found that at any temperature, polar states concentration increases monotonically with the increasing temperature at the fixed value of $U / w$ when the respective dependence in $[9,23]$ has a minimum.

The dependence of $\Delta E / U$ on parameter $U / w$ at zero temperature is plotted in figure 5. It is important to note that in the point of gap disappearence $d \neq 0$ by contrast to the previously obtained result [13]. At the increasing $U / w$, the energy gap width increases (the negative values of $\Delta E$ correspond to the overlapping of the subbands). For comparison in figure 5 the results of approximation "Hubbard-I" [7] are also plotted. In the point of energy gap vanishing $(U / w)_{\mathrm{c}}=$ 1.672 which is very close to the result of "Hubbard-III" approximation [22].

At the increase of temperature in the metallic state, the overlapping of subbands decreases and temperature induced transition from metallic to insulating state can occur at some values of parameter $U / w$ (figure 6). The dependence obtained can qualitatively explain the transitions from metallic to insulating state with the increase of temperature in the systems $\mathrm{NiS}_{2-x} \mathrm{Se}_{x},\left(\mathrm{~V}_{1-x} \mathrm{Cr}_{x}\right)_{2} \mathrm{O}_{3}$ and $\left.\mathrm{Y}_{1-x} \mathrm{Ca}_{x} \mathrm{TiO}_{3}\right)$ experimentally observed in a paramagnetic state. Note that the quantitative description of the pressuretemperature phase diagram of these compounds within the generalized Hubbard model with correlated hopping has been done in [24].

\section{Ground state energy}

The ground state energy of the model

$$
\frac{E_{0}}{N}=\frac{1}{N}\left\langle\sum_{i j \sigma} t_{i j} a_{i \sigma}^{+} a_{j \sigma}\right\rangle+U d,
$$

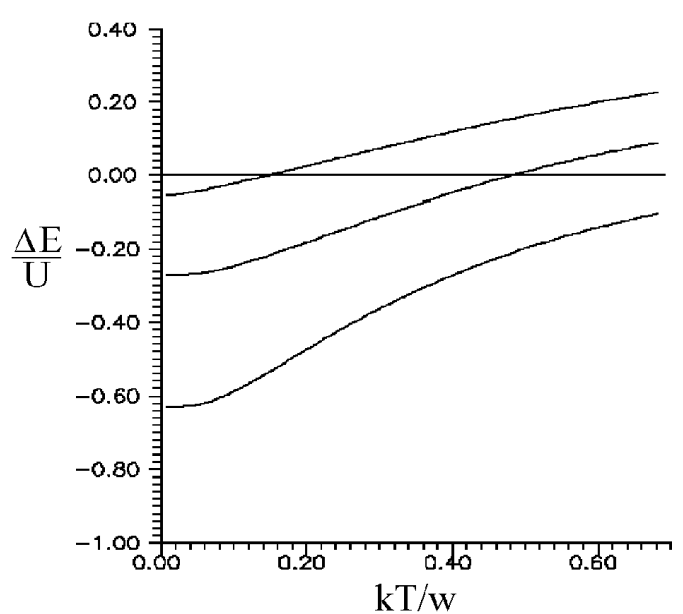

Figure 6. The dependencies of energy gap width on temperature at different $U / w$ : values of $U / w$ from down to up are $0.5,1.2,1.5$.

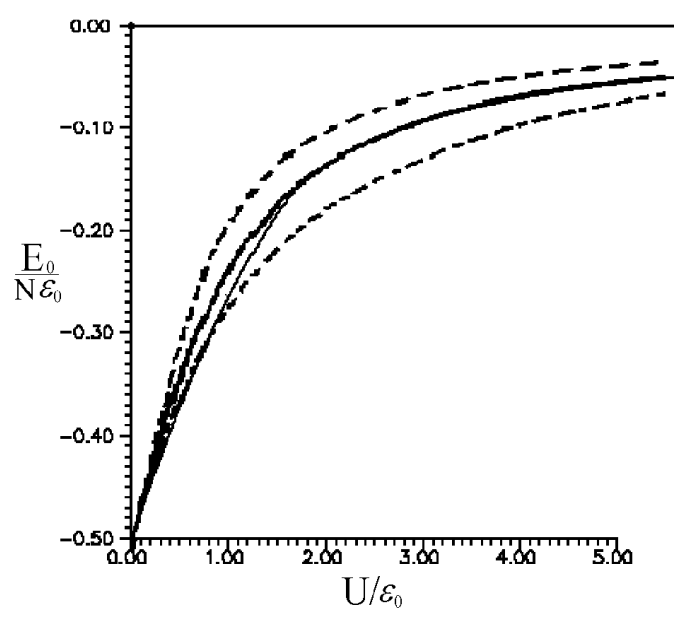

Figure 7. The comparison of ground state energies in one-dimensional case: dashed curves correspond to upper and lower bounds given by Langer and Mattis [26], upper solid curve corresponds to exact ground state (Lieb and Wu [25]) lower solid curve corresponds to the result of this paper. 


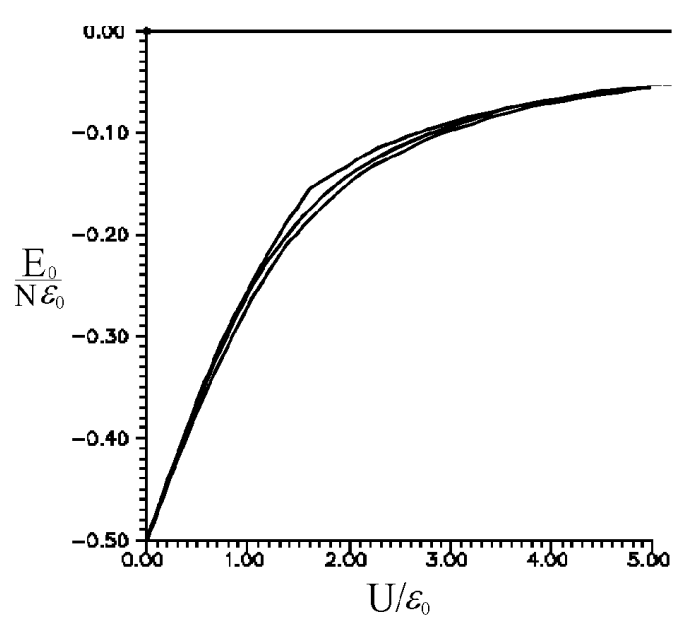

Figure 8. The ground state energy found in this paper (upper curve), best upper (middle curve) and lower (lower curve) bounds on ground state energy in infinite-dimensional case.

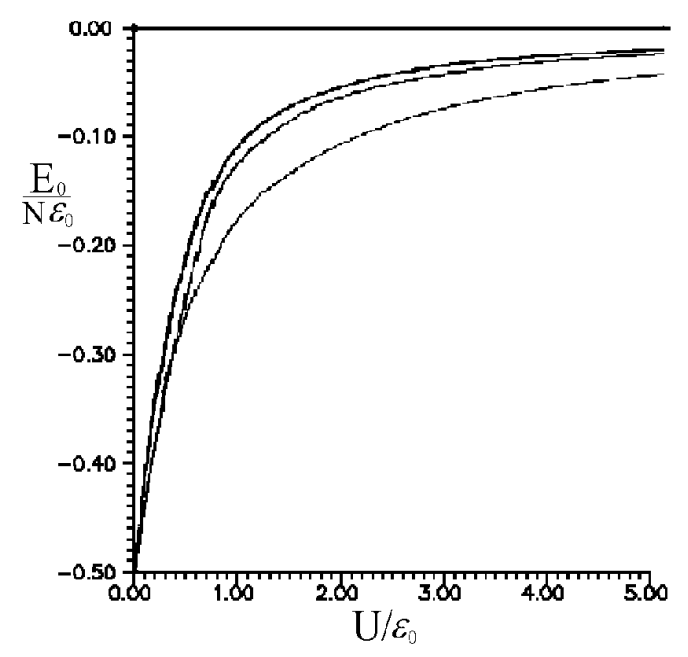

Figure 9. The upper (upper curve) and lower (lower curve) bounds on ground state energy in three-dimensional case [26] and the ground state energy found in this paper (middle curve).

calculated using single particle Green function (2.20) and expressions (3.2)-(3.3) for the concentration of polar states has the form:

$$
\frac{E_{0}}{N}=-\frac{w}{2}+\frac{U}{4}(1+3 d)-\frac{U^{2}}{2 w} \frac{(1-4 d)}{4(1-2 d)^{2}-1}
$$

if $(U / w) \leqslant(U / w)_{\mathrm{c}}$ and

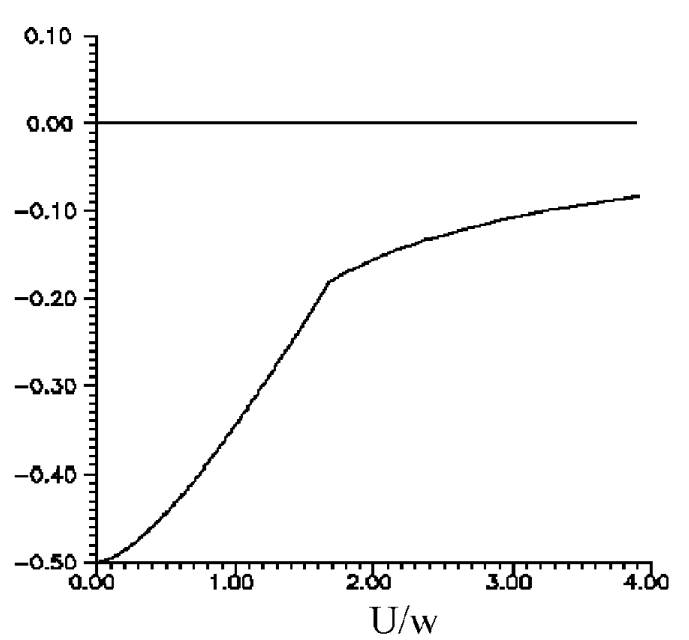

Figure 10. The kinetic part of ground state energy as a function of $U / w$.

$$
\frac{E_{0}}{N}=-\frac{1}{2} \sqrt{U^{2}+w^{2}}+2 U\left(\frac{1}{4}-d\right)
$$

if $(U / w)>(U / w)_{c}$. In figure 7 the dependence of the ground state energy on the parameter $U / w$ given by equations (4.2)(4.3) is compared with the exact result found in one-dimensional case [25]. The upper and lower bounds on ground state energy in one-dimensional case found in paper [26] are also shown. Our result for the ground state energy in a metallic state lies slightly lower than the exact one and in the insulator state it very well fits the exact ground state energy.

In figure 8 our plot of the ground state energy is compared with the best upper and lower bounds on ground state energy 
in infinite-dimensional case [27]. In figure 9 we have the comparison with bounds on ground state energy for three-dimensional simple cubic lattice obtained in paper [26]. In Figs. 7-9 the ground state energy per electron is normalized by the averaged band energy in the absence of correlation $\varepsilon_{0}$; in the considered case and rectangular density of states $\varepsilon_{0}=-w / 2$. Figs. 7-9 show that our result present a good approximation for the ground state energy of the system. In figure 10 we plot our result for the kinetic part of ground state energy. This plot describes the same behavior of kinetic energy of electrons with the change of correlation strength in a paramagnetic state like the respective result of work [23]: in a metallic state the absolute value of kinetic energy decreases rapidly due to a rapid decrease of doublon (hole) concentration. In the insulating state the absolute value of kinetic energy decreases slowly which in the approximation of the effective Hamiltonian (obtained for the case $t_{i j} / U \ll 1$ ) is equivalent to the interaction of local magnetic moments.

\section{Conclusions}

In this paper we have studied electron correlations in narrow energy bands using the recently proposed approximation [11]. We assume that the state of the narrowband system is a paramagnetic insulator or a paramagnetic metal. The single-particle Green function and the energy spectrum dependent on model parameters and on polar states concentration (thus on temperature) have been found in a paramagnetic state at half-filling $(n=1)$. The obtained expression for energy gap permits to describe MIT at the changes of bandwidth (pressure) or temperature. The comparison of the calculated ground state energy with the results of other approximations and the exact result found in one-dimensional case shows that the method used is a good approximation for the model under consideration.

It is worthwhile noting that the approximation used in this paper can be generalized to describe the effects of antiferromagnetic ordering. Such a generalization will be considered in the subsequent paper.

\section{References}

1. Mott N.F. Metal-Insulator Transitions. London, Taylor and Francis, 1990.

2. Yao X., Honig J.M., Hogan T., Kannewurf C., Spałek J. Electrical properties of $\mathrm{NiS}_{2-x} \mathrm{Se}_{x}$ single crystals: From Mott insulator to paramagnetic metal. // Phys. Rev. B, 1996, vol. 54, No. 24, p. 17469-17475; Yao X., Kuo Y.K., Powell D.K., Brill J.W., Honig J.M. Magnetic susceptibillity and heat-capacity studies of $\mathrm{NiS}_{2-x} \mathrm{Se}_{x}$ single crystals: A study of transitions at nonzero temperature. // Phys. Rev. B, 1997, vol. 56 , No. 12 , p. $7129-7135$.

3. Honig J.M., Spałek J. Electronic properties of $\mathrm{NiS}_{2-x} \mathrm{Se}_{x}$ single crystals: from magnetic Mott-Hubbard insulator to normal metals. // Chem. Mater., 1998, vol. 10, No. 10, p. 2910-2929.

4. Edvards P.P., Rao C.N.R. Metal-Insulator Transitions Revisited. London, Taylor and Francis, 1995. 
5. Taguchi Y., Tokura Y., Arima T., Inaba F. Change of electronic structure with carrier dopping in the highly correlated electron system $\mathrm{Y}_{1-x} \mathrm{Ca}_{x} \mathrm{TiO}_{3}$. // Phys. Rev. B, 1993, vol. 48 , p. $511-518$.

6. Fujimori A., Tokura Y. Spectroscopy of Mott Insulators and Correlated Metals. Berlin Heidelberg, Springer-Verlag, 1995.

7. Hubbard J. Electron correlation in narrow energy bands. // Proc. Roy. Soc. A, 1963, vol. 276, No. 1365, p. 238-257.

8. Izyumov Yu.A. The Hubbard model in the regime of strong electronic correlations. // Usp. Fiz. Nauk., 1995, vol. 165, No. 4, p. 403-427 (in Russian).

9. Georges A., Kotliar G., Krauth W., Rozenberg M. Dynamical mean-field theory of strongly correlated fermion systems and the limit of infinite dimensions. // Rev. Mod. Phys., 1996, vol. 68, No. 1, p. 13-125.

10. Gebhard F. The Mott Metal-Insulator Transition - Models and Methods. Berlin, Springer, 1997.

11. Didukh L. A new two-pole approximation in the Hubbard model. Metal-insulator transition. Preprint of Los Alamos National Laboratory, cond-mat/0002334, Los Alamos, 2000, 3 p.

12. Hubbard J. Electron correlation in narrow energy bands. The atomic representation. // Proc. Roy. Soc. A, 1965, vol. 285, No. 1403, p. 542-560.

13. Didukh L. Energy spectrum of electrons in the Hubbard model: a new mean-field approximation. // Phys. Stat. Sol. (b), 1998, vol. 206, p. R5-R6.

14. Ikeda M.A., Larsen U., Mattuck R.D. Another approach to localized magnetic moments in the Hubbard model. // Phys. Lett. A, 1972, vol. 39, p. 55-56.

15. Roth L. Electron correlations in narrow energy bands. // Phys. Rev., 1969, vol. 184, No. 2, p. 451-459.

16. Harris A.B., Lange V. Single-particle excitations in narrow energy bands. // Phys. Rev. B, 1967, vol. 157, No. 2, p. 295-314.

17. Kawabata A. One electron Green's function in magnetic insulators. // Progr. Theor. Phys., 1972, vol. 48, p. 1793-1808.

18. Beenen J., Edwards D.M. Superconductivity in the two-dimensional Hubbard model. // Phys. Rev. B, 1995, vol. 52, No. 18, p. 13636-13651.

19. Mehlig B., Eskes H., Hayn H., Meinders M.B.J. Single-particle spectral density of the Hubbard model. // Phys. Rev. B, 1995, vol. 52, No. 4, p. 2463-2470.

20. Oleś A.M., Eskes H. Two-pole approach to the Hubbard bands. // Physia B, 1995, vol. 206-207, p. 685-687.

21. Izyumov Yu.A., Chashchin N.I. Spectrum of quasiparticles in Hubbard model with strong Coulomb interaction. // Fiz. Metal. Metaloved., 1997, vol. 84, No. 6, p. 16-28 (in Russian).

22. Hubbard J. Electron correlation in narrow energy bands. An improved solution. // Proc. Roy. Soc. A, 1964, vol. 281, No. 1386, p. 401-419.

23. Kotliar G., Rozenberg M. The Mott transition in infinite dimensions: old ideas and some surprises. - In: The Hubbard Model, edited by Baeriswyl M., New-York and London, Plenum Press, 1995, p. 155-166.

24. Didukh L., Hankevych V. Pressure-temperature phase diagram of the generalized Hubbard model with correlated hopping at half-filling. Preprint of Los Alamos National Laboratory, cond-mat/0009037, Los Alamos, 2000, 7 p. (accepted for publication in Cond. Matt. Phys.). 
25. Lieb E.H., Wu F.Y. Absence of Mott transition in an exact solution of the shortrange, one-band model in one dimension. // Phys. Rev. Lett., 1968, vol. 20, No. 25, p. $1445-1448$.

26. Langer W.D., Mattis D.C. Ground state energy of Hubbard model. // Phys. Lett. A, 1971, vol. 36, p. 139-140.

27. Janiš V., Mašek J., Vollhardt D. Construction of analytically tractable mean-field theories for quantum models. // Z. Phys. B, 1993, vol. 91, p. 325-336.

\section{Електронні кореляції у вузьких енергетичних зонах: енергія основного стану та перехід метал-діелектрик}

\section{Л.Дідух, Ю.Скоренький}

Тернопільський державний технічний університет імені І. Пулюя, кафедра фізики, 46001 Тернопіль, вул. Руська, 56

Отримано 5 травня 2000 р., в остаточному вигляді 28 жовтня 2000 р.

В даній роботі електронні кореляції у вузьких енергетичних зонах вивчаються в рамках моделі Габбарда. За допомогою нового двополюсного наближення знайдено одночастинкову функцію Гріна та енергетичний спектр у парамагнітному стані при половинному заповненні. В основному стані знайдено аналітичні вирази для ширини енергетичної щілини, концентрації полярних станів та енергії системи. Досліджено переходи метал-діелектрик при зміні ширини зони та при зміні температури. Отримані результати використані для інтерпретації деяких експериментальних даних для вузькозонних матеріалів.

Ключові слова: вузькі зони провідності, гамільтоніан Габбарда, перехід метал-діелектрик, енергія основного стану

PACS: $71.28 .+d, 71.27 .+a, 71.10 . F d, 71.30 .+h$ 